\title{
Editorial
}

\section{Advances in DOA Estimation and Source Localization}

\author{
Elias Aboutanios, ${ }^{1}$ Aboulnasr Hassanien, ${ }^{2}$ Amr El-Keyi, ${ }^{3}$ \\ Youssef Nasser, ${ }^{4}$ and Sergiy A. Vorobyov ${ }^{5}$ \\ ${ }^{1}$ School of Electrical Engineering and Telecommunications, University of New South Wales, Sydney, NSW 2052, Australia \\ ${ }^{2}$ Department of Electrical Engineering, Wright State University, Dayton, OH 45435, USA \\ ${ }^{3}$ Department of Systems and Computer Engineering, Carleton University, Ottawa, ON, Canada \\ ${ }^{4}$ Department of Electrical and Computer Engineering, American University of Beirut, Beirut, Lebanon \\ ${ }^{5}$ Department of Signal Processing and Acoustics, Aalto University, 00076 Aalto, Finland \\ Correspondence should be addressed to Elias Aboutanios; elias@ieee.org
}

Received 1 August 2017; Accepted 1 August 2017; Published 27 August 2017

Copyright ( 2017 Elias Aboutanios et al. This is an open access article distributed under the Creative Commons Attribution License, which permits unrestricted use, distribution, and reproduction in any medium, provided the original work is properly cited.

Since the emergence of radars in the middle of the last century, the determination of the direction of arrival (DOA) has been an active research topic with applications in various areas including, in addition to the radar itself, sonar and communications. DOA estimation has come a long way from its early days when mechanically steered narrow-beam antennas were employed to determine the direction of incidence of the incoming signal $[1,2]$. The introduction of digital signal processors has made a wide range of mathematical techniques available for DOA estimation. Approaches such as subspace decomposition, eigenanalysis, sparsity, and compressed sensing based methods, as well as the advanced Fourier based processing techniques, are playing a fundamental role in achieving performance enhancements in speed, accuracy, and robustness.

Presently, the range of applications of DOA estimation continues to expand and the devices that rely upon it continue to proliferate. Automotive radars are currently being deployed for situational awareness, thus assisting in emergency braking, steering, and cruise control [3]. In communications, the quality of the wireless services is greatly enhanced if the user information is known. DOA estimation is, for instance, essential for the delivery of context-based and targeted information to the user [4]. In navigation, interference localization permits interferers to be disabled [5-7], which is essential to ensure safety in critical applications such as air travel.

DOA estimation is often implemented using either sensor arrays or multiple antennas working cooperatively. There exist a wide variety of approaches in the literature for obtaining the DOA of single and multiple sources. Highresolution (HR) methods and subspace-based techniques can achieve very good resolution and accuracy at the expense of high computational cost. Perhaps the best known methods are the MUSIC [8] and ESPRIT [9] algorithms, although other well-known methods include the Capon estimator [10] and matrix pencil [11]. HR approaches, however, incur a high computational cost and cannot resolve correlated sources. They also cannot be applied in the single snapshot case unless spatial smoothing is employed, which compromises both the resolution and the accuracy. In contrast, discrete Fourier transform (DFT) methods (e.g., $[12,13])$ are computationally much simpler and suitable for the single snapshot case.

Owing to the widening range of applications for DOA estimation, the increased variety of sensor configurations, and the spread of constraints imposed by the hardware (e.g., low power devices), the research into novel DOA estimation strategies has continued unabated. This special issue presents a collection of papers detailing recent advances in technologies and techniques for enhanced DOA estimation and its applications in radar, sonar, wireless communications, and other fields.

The classical maximum likelihood (ML) DOA estimation of a number of sources using an array of antennas is studied by $\mathrm{H}$. Chen et al. in the paper titled "Efficient AM Algorithms for Stochastic ML Estimation of DOA." The paper adopts an alternating minimization strategy where each source is estimated sequentially and the process is then repeated until 
all source estimates have converged. This allows the authors to reduce the computational complexity of the stochastic ML (SML) algorithm. In the paper "Robust Cyclic MUSIC Algorithm for Finding Directions in Impulsive Noise Environment," the DOA estimation problem of cyclostationary signals in impulsive noise environments is addressed. The impulsive noise is modeled using the family of alpha stable distributions that has a heavy tail. Such distributions do not have finite second-order statistics, which limits the applicability of subspace-based methods. The authors circumvent this problem by defining robust cyclic correlation functions and then employ the MUSIC algorithm to obtain the DOAs.

Reconfigurable, sparse, coprime, and conformal arrays are playing an important role in enhancing the DOA estimation performance as well. Indeed, these allow the array configuration to be used as an extra degree of freedom. In the paper titled "Experimental Results of Novel DoA Estimation Algorithms for Compact Reconfigurable Antennas," H. Paaso et al. study the problem of estimating the DOA when the antenna is reconfigurable. The authors consider a reconfigurable composite right/left handed (CRLH) leakywave antenna. They then evaluate and compare the adjacent pattern power ratio (APPR) and MUSIC algorithms for estimating the DOA. The APPR is a lookup table based approach that requires the prior characterization of the antenna.

Reduction in the number of sensors is desirable and is achieved using coprime arrays, which allow the estimation of the DOAs of more sources than sensors. A. Liu et al. deal with this problem in the paper "Direction-of-Arrival Estimation for Coprime Array Using Compressive Sensing Based Array Interpolation" by employing compressive sensing in tandem with array interpolation in order to achieve improved DOA estimation performance. Another variation in the array configuration allows the array geometry to conform to a nonflat surface, which complicates the estimation process. The paper "DOA Estimation of Cylindrical Conformal Array Based on Geometric Algebra" by M. Wu et al. addresses the DOA estimation for conformal arrays by combining a geometric algebra approach with MUSIC. The authors propose the GAMUSIC algorithm which simplifies the calculations.

Antenna arrays provide multiple multidimensional data, permitting the estimation of multiple spatial parameters such as azimuth and elevation as well as temporal parameters, such as range and Doppler. Therefore, two- and multidimensional data processing techniques play an enabling role in source parameter estimation. In the paper "Azimuth/Elevation Directional Finding with Automatic Pair Matching," N. Tayem deals with the problem of two-dimensional (2D) DOA estimation for multiple far-field sources. The azimuth and elevation angles are obtained via constructing three crosscorrelation matrices and applying parallel factor analysis. Automatic pair matching is achieved via using trilinear least squares based solutions. In the paper "Performance Analysis of Two-Dimensional Maximum Likelihood Direction-ofArrival Estimation Algorithm," on the other hand, Y.-S. Cho et al. consider the $2 \mathrm{D}$ problem of estimating the azimuth and elevation using a uniform circular array (UCA). More specifically, the authors study the ML estimator and derive explicit expressions for the mean squared error.
Higher dimensional data comprising, for instance, space, time, frequency, and polarization is studied in the paper "Tensor-Based Methods for Blind Spatial Signature Estimation in Multidimensional Sensor Arrays." The work presents two tensor-based techniques that employ the covariance tensor to obtain the DOAs. The first method assumes that the sources are correlated and hence the covariance matrix is not diagonal. In the second case, the sources are assumed to be uncorrelated and the method is shown to be equivalent to parallel factor analysis (PARAFAC). The DOA estimation is obtained using an alternating least squares (ALS) approach.

DOA estimation finds an interesting application in the paper "Application Research of the Sparse Representation of Eigenvector on the PD Positioning in the Transformer Oil." In this work, the authors consider the problem of detecting partial discharge in high voltage transformer oil where the ultrasonic signal is wideband. Using a circular array, the signal is spatially sampled and then DOA estimation is implemented using sparse decomposition and the matching pursuit algorithm.

The works presented in this special issue showcase the depth of the DOA estimation problem as well as the range of challenges and breadth of applications. We hope that the contributions made by the published papers will advance the state of the art and contribute to this fertile field.

\section{Acknowledgments}

We would like to thank the authors for their novel and insightful contributions and the anonymous reviewers for their positive and helpful feedback.

Elias Aboutanios
Aboulnasr Hassanien
Amr El-Keyi
Youssef Nasser
Sergiy A. Vorobyov

\section{References}

[1] H. Krim and M. Viberg, "Two decades of array signal processing research," IEEE Signal Processing Magazine, vol. 13, no. 4, pp. 6794, 1996.

[2] T. E. Tuncer and B. Friedlander, Eds., Classical and Modern Direction-of-Arrival Estimation, Academic Press, 2009.

[3] F. Engels, P. Heidenreich, A. M. Zoubir, F. K. Jondral, and M. Wintermantel, "Advances in automotive radar: a framework on computationally efficient high-resolution frequency estimation," IEEE Signal Processing Magazine, vol. 34, no. 2, pp. 36-46, 2017.

[4] A. Yassin, Y. Nasser, M. Awad et al., "Recent advances in indoor localization: a survey on theoretical approaches and applications," IEEE Communications Surveys \& Tutorials, vol. 19, no. 2, pp. 1327-1346, 2017.

[5] X. Wang, M. Amin, F. Ahmad, and E. Aboutanios, "Interference DOA estimation and suppression for GNSS receivers using fully augmentable arrays," IET Radar, Sonar \& Navigation, vol. 11, no. 3, pp. 474-480, 2017.

[6] M. G. Amin, X. Wang, Y. D. Zhang, F. Ahmad, and E. Aboutanios, "Sparse arrays and sampling for interference mitigation and 
DOA estimation in GNSS," Proceedings of the IEEE, vol. 104, no. 6, pp. 1302-1317, 2016.

[7] W. Abdessamad, Y. Nasser, H. Artail, S. Chazbek, G. Fakher, and O. Bazzi, "An SDR platform using direction finding and statistical analysis for the detection of interferers," in Proceedings of the 8th International Congress on Ultra Modern Telecommunications and Control Systems and Workshops (ICUMT '16), pp. 43-48, Lisbon, Portugal, October 2016.

[8] R. O. Schmidt, "Multiple emitter location and signal parameter estimation," IEEE Transactions on Antennas and Propagation, vol. 34, no. 3, pp. 276-280, 1986.

[9] R. Roy, A. Paulraj, and T. Kailath, "ESPRIT—a subspace rotation approach to estimation of parameters of cisoids in noise," IEEE Transactions on Acoustics, Speech, and Signal Processing, vol. 34, no. 5, pp. 1340-1342, 1986.

[10] J. Capon, "High-resolution frequency-wavenumber spectrum analysis," Proceedings of the IEEE, vol. 57, no. 8, pp. 1408-1418, 1969.

[11] Y. Hua and T. K. Sarkar, "Matrix pencil method for estimating parameters of exponentially damped/undamped sinusoids in noise," IEEE Transactions on Acoustics, Speech, and Signal Processing, vol. 38, no. 5, pp. 814-824, 1990.

[12] J. Li and P. Stoica, "Efficient mixed-spectrum estimation with applications to target feature extraction," IEEE Transactions on Signal Processing, vol. 44, no. 2, pp. 281-295, 1996.

[13] E. Aboutanios, A. Hassanien, M. G. Amin, and A. M. Zoubir, "Fast iterative interpolated beamforming for accurate singlesnapshot DOA estimation," IEEE Geoscience and Remote Sensing Letters, vol. 14, no. 4, pp. 574-578, 2017. 


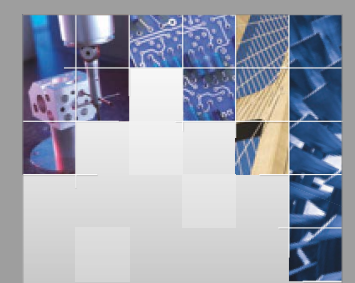

\section{Enfincering}
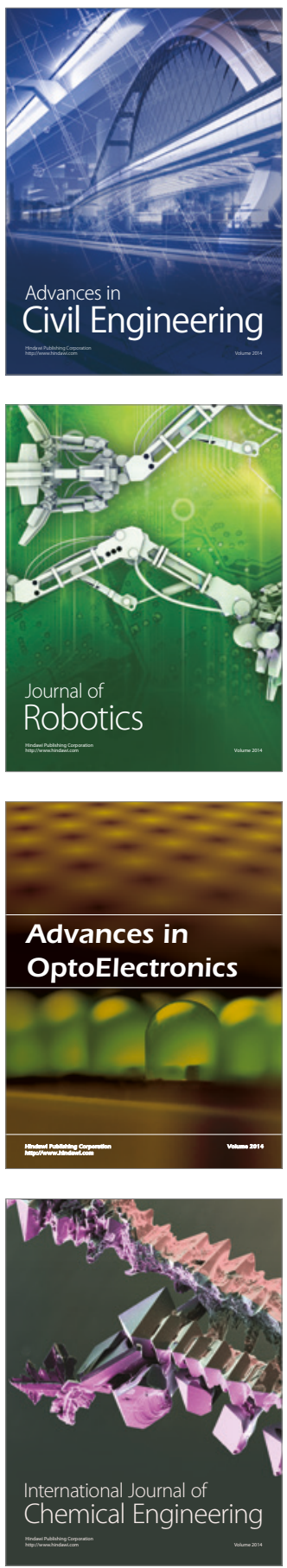

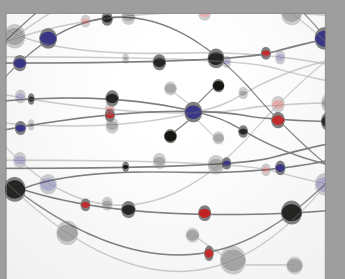

The Scientific World Journal

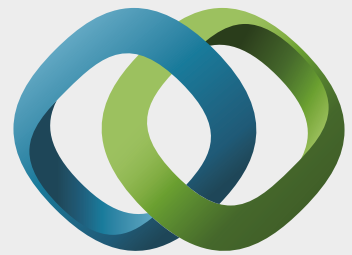

\section{Hindawi}

Submit your manuscripts at

https://www.hindawi.com
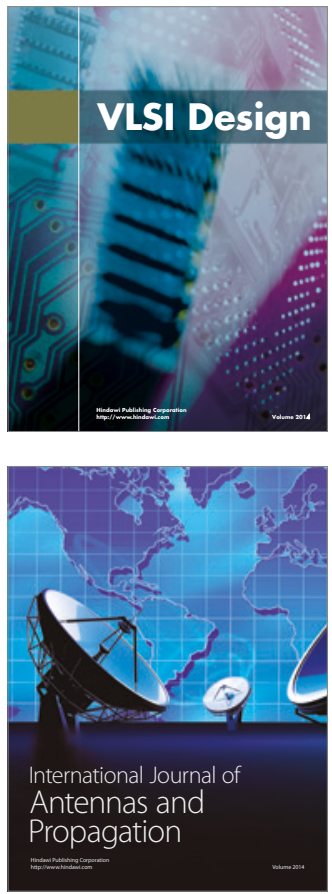

\section{Rotating}

Machinery
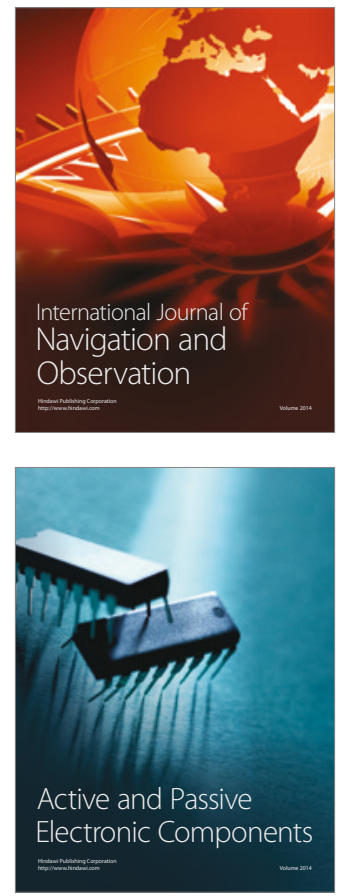
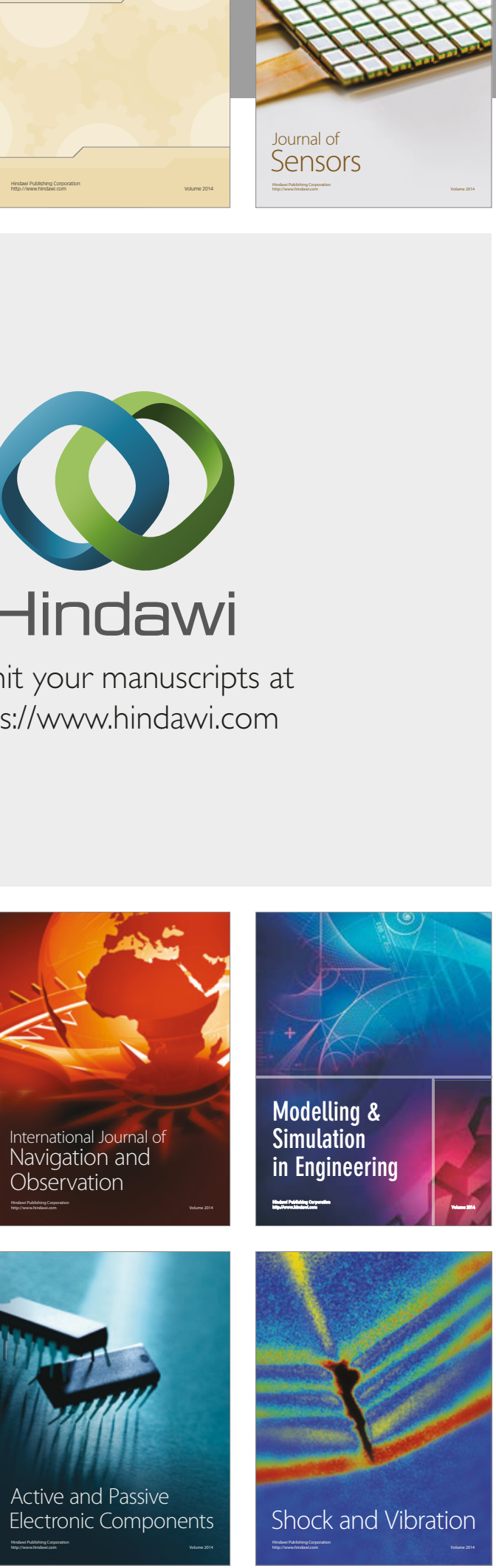
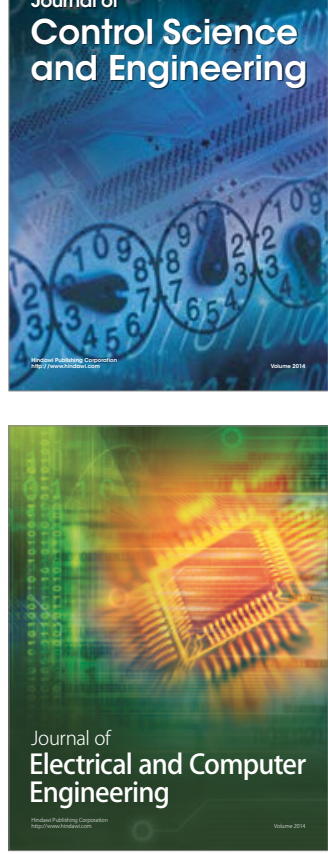

Distributed

Journal of

Control Science

and Engineering
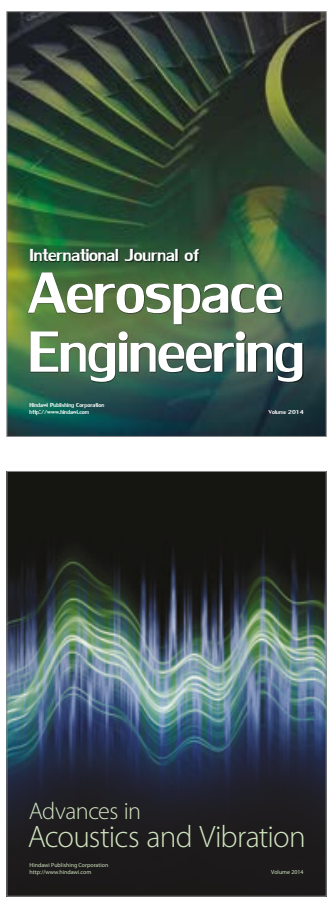

Sensor Networks 